\title{
Article \\ Overexpression of an Osa-miR162a Derivative in Rice Confers Cross-Kingdom RNA Interference-Mediated Brown Planthopper Resistance without Perturbing Host Development
}

\author{
Wenzhong Shen ${ }^{1} \oplus$, Shanni Cao ${ }^{1}$, Jinhui Liu ${ }^{1}$, Wenqing Zhang ${ }^{1}$, Jie Chen ${ }^{2, *}$ and Jian-Feng Li ${ }^{1, *} \mathbb{C}$ \\ 1 State Key Laboratory of Biocontrol, Guangdong Provincial Key Laboratory of Plant Resources, \\ School of Life Sciences, Sun Yat-sen University, Guangzhou 510275, China; \\ shenwzh5@mail.sysu.edu.cn (W.S.); caoshn@mail2.sysu.edu.cn (S.C.); liujh89@mail2.sysu.edu.cn (J.L.); \\ lsszwq@mail.sysu.edu.cn (W.Z.) \\ 2 Guangdong Provincial Key Laboratory of High Technology for Plant Protection, \\ Plant Protection Research Institute, Guangdong Academy of Agricultural Sciences, \\ Guangzhou 510640, China \\ * Correspondence: chenj@gdppri.com (J.C.); lijfeng3@mail.sysu.edu.cn (J.-F.L.); \\ Tel./Fax: +86-20-39943513 (J.-F.L.)
}

\section{check for} updates

Citation: Shen, W.; Cao, S.; Liu, J.; Zhang, W.; Chen, J.; Li, J.-F. Overexpression of an Osa-miR162a Derivative in Rice Confers Cross-Kingdom RNA Interference-Mediated Brown Planthopper Resistance without Perturbing Host Development. Int. J. Mol. Sci. 2021, 22, 12652. https:// doi.org/10.3390/ijms222312652

Academic Editor: Elisabeth Jamet

Received: 20 October 2021

Accepted: 22 November 2021

Published: 23 November 2021

Publisher's Note: MDPI stays neutra with regard to jurisdictional claims in published maps and institutional affiliations.

Copyright: (c) 2021 by the authors. Licensee MDPI, Basel, Switzerland. This article is an open access article distributed under the terms and conditions of the Creative Commons Attribution (CC BY) license (https:// creativecommons.org/licenses/by/ $4.0 /)$.
Abstract: Rice is a main food crop for more than half of the global population. The brown planthopper (BPH, Nilaparvata lugens) is one of the most destructive insect pests of rice. Currently, repeated overuse of chemical insecticides represents a common practice in agriculture for BPH control, which can induce insect tolerance and provoke environmental concerns. This situation calls for innovative and widely applicable strategies for rice protection against $\mathrm{BPH}$. Here we report that the rice osamiR162a can mediate cross-kingdom RNA interference (RNAi) by targeting the NITOR (Target of rapamycin) gene of $\mathrm{BPH}$ that regulates the reproduction process. Through artificial diet or injection, osa-miR162a mimics repressed the NITOR expression and impaired the oviposition of BPH adults. Consistently, overproduced osa-miR162a in transgenic rice plants compromised the fecundity of BPH adults fed with these plants, but meanwhile perturbed root and grain development. To circumvent this issue, we generated osa-miR162a-m1, a sequence-optimized osa-miR162a, by decreasing base complementarity to rice endogenous target genes while increasing base complementarity to NITOR . Transgenic overexpression of osa-miR162a-m1 conferred rice resistance to $\mathrm{BPH}$ without detectable developmental penalty. This work reveals the first cross-kingdom RNAi mechanism in rice-BPH interactions and inspires a potentially useful approach for improving rice resistance to $\mathrm{BPH}$. We also introduce an effective strategy to uncouple unwanted host developmental perturbation from desirable cross-kingdom RNAi benefits for overexpressed plant miRNAs.

Keywords: brown planthopper; cross-kingdom RNA interference; NITOR; osa-miR162a; rice

\section{Introduction}

Rice (Oryza sativa) is one of the most important staple food crops worldwide. The brown planthopper (BPH, Nilaparvata lugens) is an infamous rice-specific pest that has plagued numerous times in history and caused enormous losses of rice production [1]. In the past years, the application of excessive chemical insecticides remains a prevailing strategy for controlling this pest, at the expense of environmental damage and induced insecticide adaptation of the pests [2]. Another valuable and widely adoptable pest control strategy is the use of transgenic crops that produce the insecticidal Cry toxins from Bacillus thuringiensis (Bt) [3]. Unfortunately, Bt crops turned out to be ineffective for controlling piercing-sucking insects, such as $\mathrm{BPH}$, which have developed a high-level resistance to a wide range of traditional pesticides [4,5]. Therefore, novel insecticidal transgene resources urgently need to be explored for rice protection against $\mathrm{BPH}$. 
MicroRNAs (miRNAs) have been demonstrated to play vital roles in regulating the development and metamorphosis of insects [6-10]. In BPH, disruption of Dicer-1, which encodes a key enzyme in miRNA biosynthesis, substantially impaired the ovarian development and fecundity [11], while the miR-4868b of BPH also regulated the fecundity by targeting the NlGS (Glutamate synthase) gene [12]. Moreover, two conserved miRNAs, miR-8-5p and miR-2a-3p, modulated chitin biosynthesis in response to the ecdysterone signaling [13]. In addition, the miR-2703 and miR-173 of BPH were found to regulate the molting metamorphosis by targeting the NlCHSA (Chitin synthase gene A) or Ftz-F1 (Fushi tarazu-F1) gene, respectively [14,15]. Notably, miRNAs regulating insect development can also come from plants through food intake. For instance, an insect chitinasetargeting miRNA expressed by transgenic tobacco plants arrested the molting process of the insect larva fed with these plants [16]. More interestingly, a phenomenon called the cross-kingdom RNA interference (RNAi) has been observed during plant-insect interactions, wherein certain plant endogenous miRNAs can be ingested when the insects feed on plant food sources. Subsequently, these plant miRNAs pass through the midgut, enter the hemolymph, accumulate in various tissues, and modulate insect gene expression. For example, the mulberry miRNAs were found to be present in the hemolymph and several tissues of silkworms [17], while plant miR162a from pollens affected the caste formation of honeybees by inhibiting the expression of the Target of rapamycin (TOR) gene [18]. More generally, plant miRNAs have been shown to enter plant-interacting fungi or mammals to induce cross-kingdom RNAi [19-21]. Based on these findings, it is tempting to assume that certain rice miRNA might be able to enter $\mathrm{BPH}$ to interfere with the development of the pest. Such knowledge may inspire new insecticidal strategies for BPH management.

Recently, the roles of rice miRNAs in regulating BPH resistance have begun to be appreciated. The osa-miR156 of rice has been demonstrated to negatively regulate $\mathrm{BPH}$ resistance, as silencing of osa-miR156 enhanced rice resistance to BPH [22]. The osamiR396 also negatively regulated $\mathrm{BPH}$ resistance in rice through the target gene OsGRF8 (Growth regulating factor 8), which positively modulated the BPH-responsive expression of flavanone 3-hydroxylase. Silencing of osa-miR396 increased flavonoid accumulation and rice resistance to $\mathrm{BPH}$ [23]. Of note, thus far no rice miRNA has been identified to promote $\mathrm{BPH}$ resistance, let alone enter $\mathrm{BPH}$ to reduce the fitness of the pest.

The biosynthesis and accumulation of vitellogenin $(\mathrm{Vg})$ in developing oocytes is of great importance for insect reproduction [24-26]. The expression of $V g$ is controlled by the TOR signaling pathway [27-30]. In BPH female adults with silenced NITOR expression, the transcript level of $V g$ was reduced, which was followed by the inhibition of the ovarian development [31,32]. The decrease of NITOR expression also resulted in retarded development of the accessory gland in BPH male adults [33]. Moreover, inhibition of TOR expression could compromise the ovarian development in mosquitoes [34].

In this study, we hypothesized and verified that rice osa-miR162a could silence the NITOR expression in BPH via the cross-kingdom RNAi and impair the fecundity of the insects. Overexpression of osa-miR162a led to augmented rice resistance in BPH, but meanwhile influenced root and grain development due to the perturbation of rice endogenous gene expression. To deal with this problem, we overexpressed an optimized osa-miR162a derivative, which harbors increased mismatches to rice endogenous target genes but decreased mismatches to NITOR. These efforts allowed us to retain the BPH resistance in transgenic overexpression plants but relieve the adverse impact of osa-miR162a overexpression on rice development.

\section{Results}

\subsection{The NITOR Gene of BPH Is a Target of Rice Osa-miR162a}

Recently, the cole crop (Brassica campestris) bra-miR162a has been reported to enter the honeybee (Apis mellifera) to target and silence the AmTOR gene via the cross-kingdom RNAi [18]. As plant miR162 exhibits a remarkable conservation across diverse species of dicots and monocots (Figure 1A) and the insect TOR gene also shows a high similarity in 
sequence and function $[28,35]$, we hypothesized that the NITOR gene of BPH may also be targeted by rice osa-miR162a, which has an identical sequence to bra-miR162a (Figure 1A). In support of this hypothesis, two bioinformatic algorithms, RNAhybrid and miRanda, predicted a primary target site for osa-miR162a in the coding sequence (CDS) of NITOR with 14 bases matching and a secondary target site in the $3^{\prime}$ untranslated region (UTR) with 10 bases matching (Figure 1B).

A

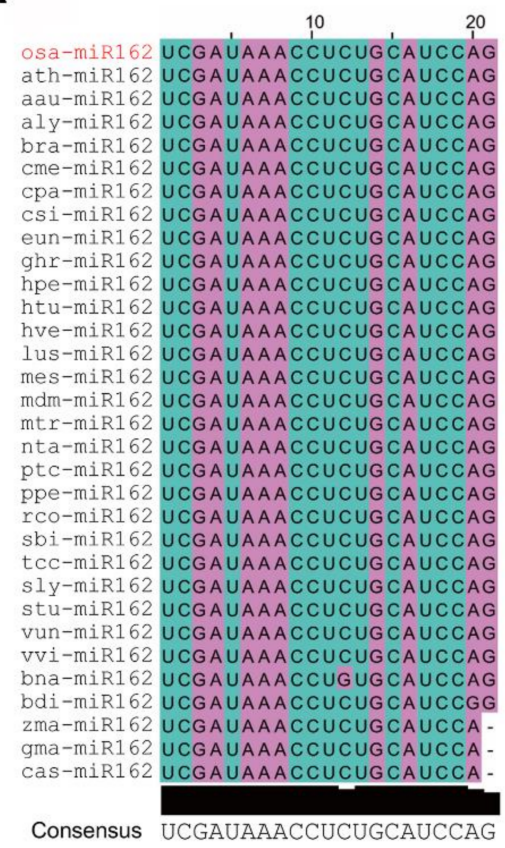

B

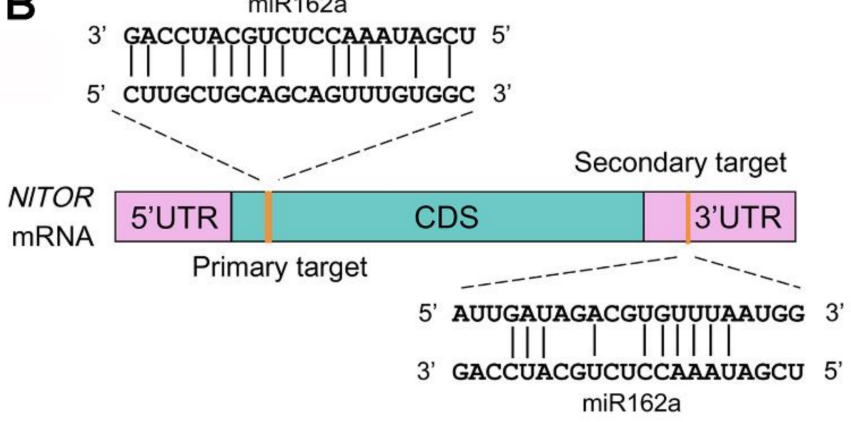

C

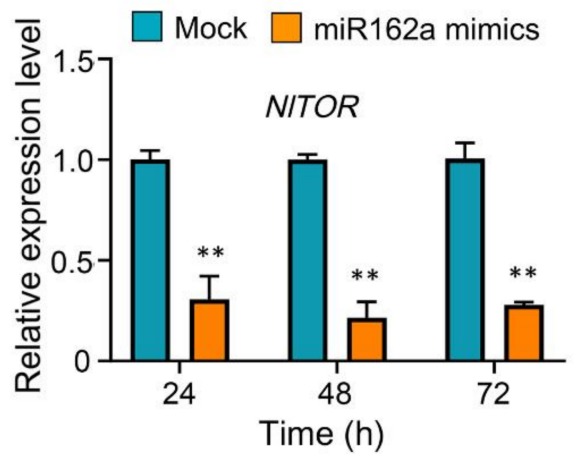

Figure 1. The NITOR gene of BPH is a target of rice osa-miR162a. (A) Sequence alignment indicates that miR162 is highly conserved across 32 plant species. The listed plant miR162 sequences were obtained from the miRBase database. (B) Putative target sites of osa-miR162 in NITOR. The target sites are indicated by orange sticks. CDS, coding sequence; UTR, untranslated region. (C) NITOR expression decreases in BPH female adults after injection with osa-miR162a mimics. The expression levels were quantified by RT-qPCR and the data are presented as mean \pm SEM of three biological replicates $(n=3)$. The expression levels of NITOR after injection with negative control miRNA mimics at different time points were normalized as $1 .{ }^{* *} p<0.01$ (student's $t$-test).

To quickly evaluate the possibility of cross-kingdom RNAi by rice osa-miR162a in $\mathrm{BPH}$, we examined whether food intake enables osa-miR162a to enter BPH, pass through the midgut, and spread to other tissues. To this end, ten BPH female adults of the first day were fed with artificial diets supplemented with osa-miR162a mimics. After BPH feeding for 24,48 , and $72 \mathrm{~h}$, the presence of osa-miR162a in different tissues was monitored by stem-loop quantitative reverse transcription PCR (RT-qPCR) [36]. The results showed that osa-miR162a could be highly accumulated in several insect tissues, such as midgut, fat body, and hemolymph after $72 \mathrm{~h}$ of feeding (Figure S1A). The presence of osa-miR162a inside BPH was further validated by Sanger sequencing of the mixed PCR products from the above tissues (Figure S1B). These results hinted that osa-miR162a is able to pass through the midgut and accumulate in other tissues of BPH through food intake. Next, the BPH female adults of the first day were injected with chemically pure osa-miR162a mimics and the expression level of NITOR was evaluated by RT-qPCR in a time course manner. At 24, 48 , and $72 \mathrm{~h}$ post injection, the transcript levels of NITOR declined dramatically relative to mock treatment (Figure 1C), suggesting that NITOR can be targeted by osa-miR162a in $\mathrm{BPH}$. 


\subsection{Injected Osa-miR162a Mimics Reduce the Fecundity of BPH}

$\mathrm{Vg}$ is a frequently used molecular marker for assessing the fecundity of BPH [29], which is regulated by the TOR signaling pathway through the downstream gene NIS6K (Ribosomal S6 kinase 1) [31] (Figure 2A). The BPH female adults of the first day were injected with osa-miR162a mimics to examine the effects on expression levels of NlS6K and $\mathrm{NlVg}$. RT-qPCR results showed that the transcript level of NlS6K decreased markedly at 24, 48, and $72 \mathrm{~h}$ after injection (Figure 2B), while that of $\mathrm{NlVg}$ declined at 24 and $48 \mathrm{~h}$ after injection but was recovered at $72 \mathrm{~h}$ (Figure 2C), probably due to compensatory regulation of $V g$ expression by other pathways in BPH under the experimental conditions. Nevertheless, after osa-miR162a mimics were injected into newly emerged female adults, there was a significant reduction in oviposition for insects injected with osa-miR162a mimics relative to those with mock treatment (Figure 2D). Together, these results implied that osa-miR162a can reduce the fecundity of $\mathrm{BPH}$ through disturbing the TOR signaling pathway.

A

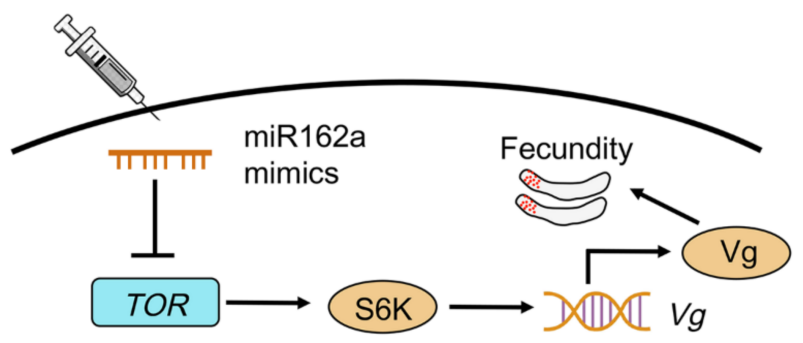

B

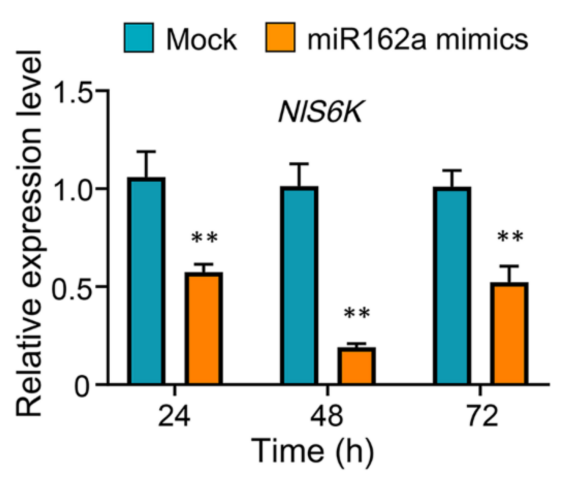

C

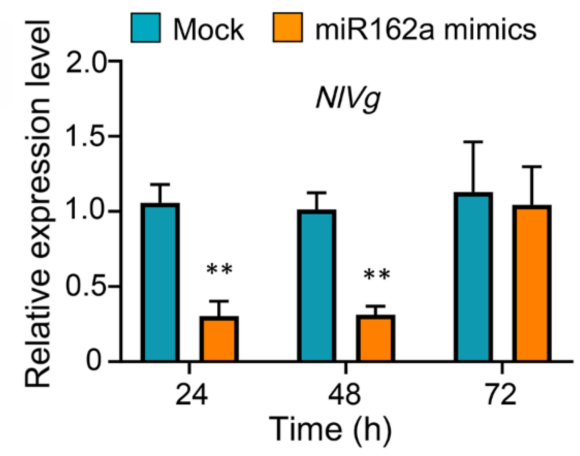

D

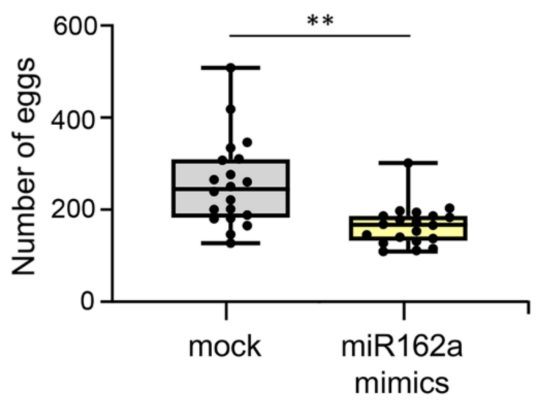

Figure 2. Injected osa-miR162a mimics impair the fecundity of BPH. (A) Schematic diagram of the effect of injected osa-miR162a mimics on weakening the TOR signaling-regulated fecundity in BPH. S6K is a downstream player of NITOR in the TOR signaling and vitellogenin $(\mathrm{Vg})$ is a molecular marker for assessing the fecundity of BPH. (B,C) Expression of NlS6K (B) and $N l V g(C)$ is reduced in female adults injected with osa-miR162a mimics. The expression levels were quantified by RT-qPCR and the data are presented as mean \pm SEM of three biological replicates $(n=3)$. The expression levels of NlS6K and $N l V g$ after injection with negative control miRNA mimics at different time points were normalized as 1. (D) Number of eggs per female adult in the fecundity assay. Eggs from 20 female adults were counted and individual data points are shown as dots. ${ }^{* *} p<0.01$ (student's $t$-test). 


\subsection{Osa-miR162a-m1 Exhibits Minimal Perturbation on Rice Endogenous Gene Expression}

The NITOR gene plays essential roles in governing the development, reproduction, and nutrient sensing of $\mathrm{BPH}[32,33]$. Based on the aforementioned observations, we reasoned that overexpression of osa-miR162a in transgenic rice plants may be able to protect rice from BPH attacks by silencing NITOR via the cross-kingdom RNAi. However, we noted that there are eight rice genes predicted to be targeted by osa-miR162a (Figure 3A,B), suggesting that overexpression of osa-miR162a would affect the expression of these rice genes as well. To overcome this problem, we decided to modify the sequence of osamiR162a to achieve the following goals: (1) increase its mismatches to putative rice target genes to minimize the silencing of those genes; (2) reduce the mismatches to the NITOR gene to maintain or even strengthen the cross-kingdom RNAi. After screening a number of rationally designed osa-miR162a derivatives using the Web MicroRNA Designer (WMD), a candidate named osa-miR162a modified 1 (osa-miR162a-m1) was selected for two reasons: firstly, no rice gene was predicted to be targeted by osa-miR162a-m1 (Figure 3A); secondly, osa-miR162a-m1 possesses two more base matches to the primary target site of NITOR than osa-miR162a (Figure 3C), thus being potentially more effective in silencing NITOR than the native osa-miR162a.

Next, we generated transgenic rice plants overexpressing osa-miR162a or osa-miR162a$\mathrm{m} 1$ in the ZH11 (Zhonghua 11) background. The accumulation of mature osa-miR162a or osa-miR162a-m1 in the T1 transgenic plants was evaluated using stem-loop RT-qPCR. Multiple osa-miR162a overexpression (miR162a-OE) lines and osa-miR162a-m1 overexpression (miR162a-m1-OE) lines exhibited readily detectable production of osa-miR162a or osa-miR162a-m1, respectively (Figure S2). The miR162a-OE lines 5 and 10 and miR162a-m1$O E$ lines 1 and 3 were selected for further analysis due to their high expression levels and overall wild-type appearance.

To examine the effect of osa-miR162a or osa-miR162a-m1 overexpression on putative target genes in rice, we checked the transcript levels of these genes in the leaf sheaths of the selected transgenic lines. Six of the eight genes, namely LOC_Os07g08500, LOC_Os05g03000, LOC_Os01g13300, LOC_Os02g39080, LOC_Os07g24400, and LOC_Os03g02970 (OsDCL1, Dicer-like 1), were strongly down-regulated in the two miR162a-OE lines relative to ZH11 plants (Figure 3D). By contrast, none of the eight genes, except OsDCL1, was repressed in miR162a-m1-OE lines (Figure 3D). The silencing of OsDCL1 by osa-miR162a-m1 was completely unexpected, as this gene has not been predicted as a target gene of osa-miR162a$\mathrm{m} 1$ by WMD due to the presence of six mismatches (Figure S3). Nevertheless, the above results suggested that osa-miR162a-m1, upon overexpression, has minimal perturbation on rice endogenous gene expression.

\subsection{Rice Development Is Not Defected upon Osa-miR162a-m1 Overexpression}

We further assessed the effect of osa-miR162a or osa-miR162a-m1 overexpression on rice growth and development. Although the heights and tiller numbers of one-month-old plants showed no significant difference between ZH11, miR162a-OE, and miR162a-m1-OE plants (Figure S4A-C), we noted that both the grain widths and grain weights of miR162a$O E$ lines were significantly reduced relative to ZH11 plants (Figure $4 \mathrm{~A}-\mathrm{C}$ ). In agreement with these results, a recent study also reported that overproduction of osa-miR162a in transgenic rice plants could generate narrower and lighter grains [37]. Of note, the grain abnormality was not observed in miR162a-m1-OE lines (Figure 4A-C). In addition, we noticed that the miR162a-OE lines also exhibited compromised root development at the early developmental stage. The three-day-old miR162a-OE seedlings had significantly shorter primary roots than ZH11 plantlets (Figure 4D,E), while the seven-day-old miR162a-OE seedlings showed slightly reduced crown root numbers relative to ZH11 plantlets (Figure 4F,G). By contrast, there was no obvious difference between miR162a-m1-OE lines and ZH11 plants in root development (Figure $4 \mathrm{D}-\mathrm{G}$ ). These results indicated that overexpression of osa-miR162a-m1, unlike osa-miR162a, exerts no adverse effect on rice development. 
A

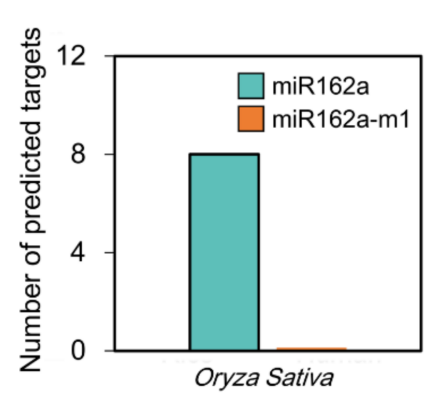

C

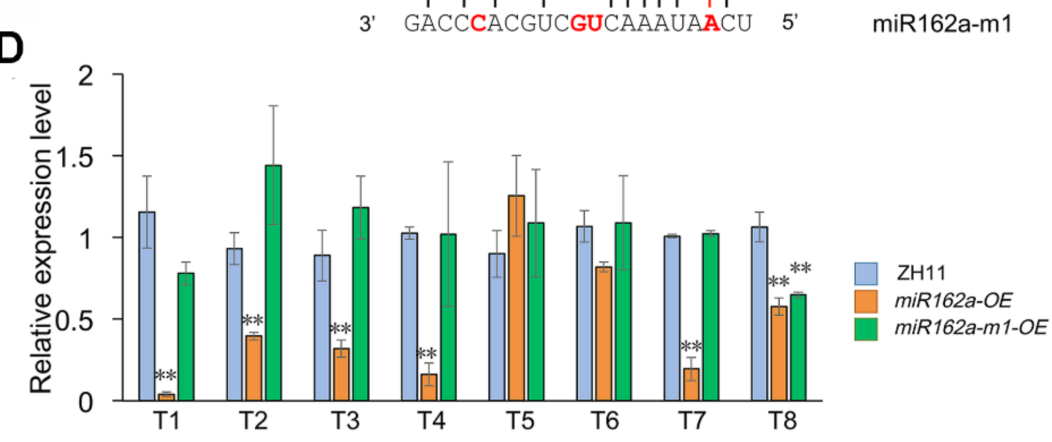

B

\begin{tabular}{cc}
\hline $\begin{array}{c}\text { miR162a } \\
\text { target }\end{array}$ & $\begin{array}{c}\text { Gene } \\
\text { ID }\end{array}$ \\
\hline $\mathrm{T} 1$ & LOC_Os07g08500 \\
$\mathrm{T} 2$ & LOC_Os05g03000 \\
$\mathrm{T} 3$ & LOC_Os01913300 \\
$\mathrm{T} 4$ & LOC_Os02939080 \\
$\mathrm{T} 5$ & LOC_Os12g11190 \\
$\mathrm{T} 6$ & LOC_Os04g49780 \\
$\mathrm{T} 7$ & LOC_Os07g24400 \\
$\mathrm{T} 8$ & LOC_Os03g02970 \\
\hline
\end{tabular}

Seed region

3' GACCUACGUCUCCAAAUAGCU 5' miR162a 5'-ACGCCUUGCUGCAGCAGUUUGUGGCUGGA-3' NITOR

3' GACCCACGUCGUCAAAUAACU 5' miR162a-m1

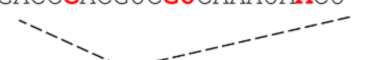

Secondary target

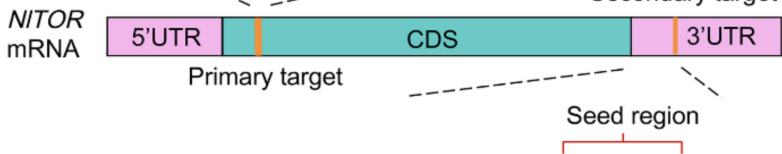
3. GACCUACGUCUCCAAAUAGCU $5{ }^{\prime}$ miR162a 5'-TGAGTAUUGAYGAGCGUGUYUAAUGGTTCC -3' NITOR

3' GACCCACGUCGUCAAAUAACU 5 , miR162a-m1

Figure 3. osa-miR162a-m1 exhibits minimal perturbation on rice endogenous gene expression. (A) Predicted target gene numbers of osa-miR162a and its derivative osa-miR162a-m1 by WMD. (B) Predicted rice target genes of osa-miR162a by WMD. (C) osa-miR162a-m1 exhibits additional base matches to the primary target site of NITOR and improved complementarity to the seed region of the secondary target site of NITOR relative to osa-miR162a. The modified nucleotides and newly gained base pairings in osa-miR162a-m1 are highlighted in red. (D) Overexpression (OE) of osa-miR162a-m1 exhibits much less perturbation on rice endogenous gene expression than osa-miR162a. The expression levels of putative target genes were determined by RT-qPCR and the data are shown as mean $\pm \mathrm{SD}$ of three biological replicates $(n=3) .{ }^{* *} p<0.01$ (Student's $t$-test).

\subsection{BPH Adults Fed with miR162a-m1-OE Plants Display Impaired Fecundity}

As we have shown that injected osa-miR162a mimics could silence the NITOR expression in BPH (Figure 1C) and compromise the oviposition of female insects (Figure 2D), we investigated whether the fecundity of $\mathrm{BPH}$ could be affected by continuous feeding on miR162a-OE or miR162a-m1-OE plants. To this aim, a pair of BPH female adult and male adult were fed with the same rice plant and were mated before oviposition. We found that more black eggs were laid by female adults fed with miR162a-OE or miR162a-m1-OE plants relative to ZH11 plants (Figure 5A). In addition, many eggs from BPH adults fed with miR162a-OE or miR162a-m1-OE plants failed to accumulate pigments in the eyes, a sign of abnormal egg development, whereas those from BPH adults fed with ZH11 plants exhibited normally pigmented eyes (Figure 5A). Moreover, the insects fed with miR162a-OE or miR162a-m1-OE plants produced slightly fewer eggs than those fed with 
ZH11 plants (Figure 5B). Furthermore, the hatching rates of eggs laid by female adults fed with miR162a-OE or miR162a-m1-OE plants were significantly lower than those fed with ZH11 plants (Figure 5C).

A

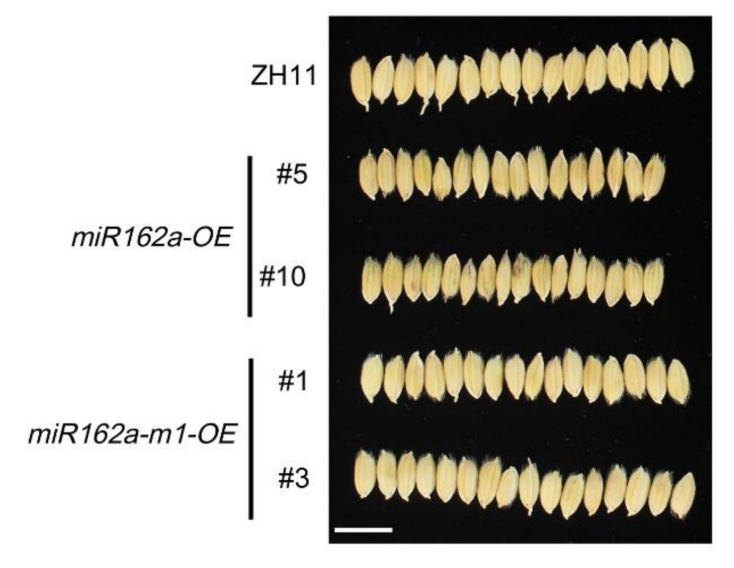

D
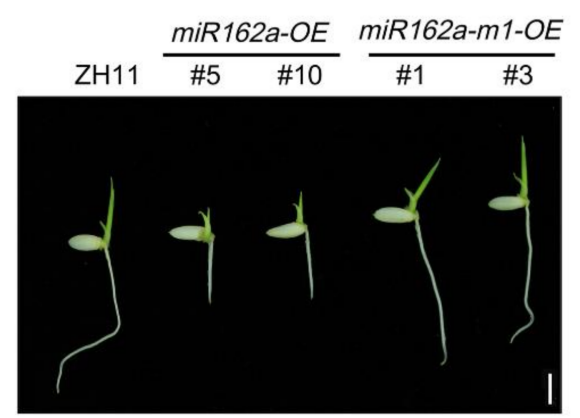

$\mathbf{F}$

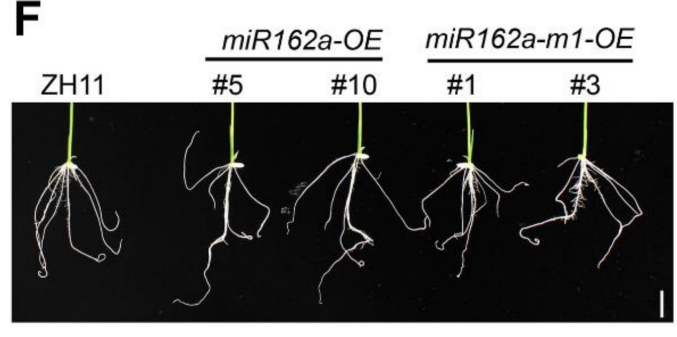

B

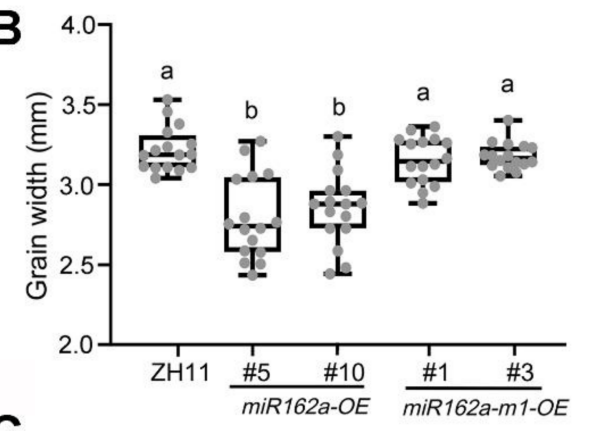

C

E
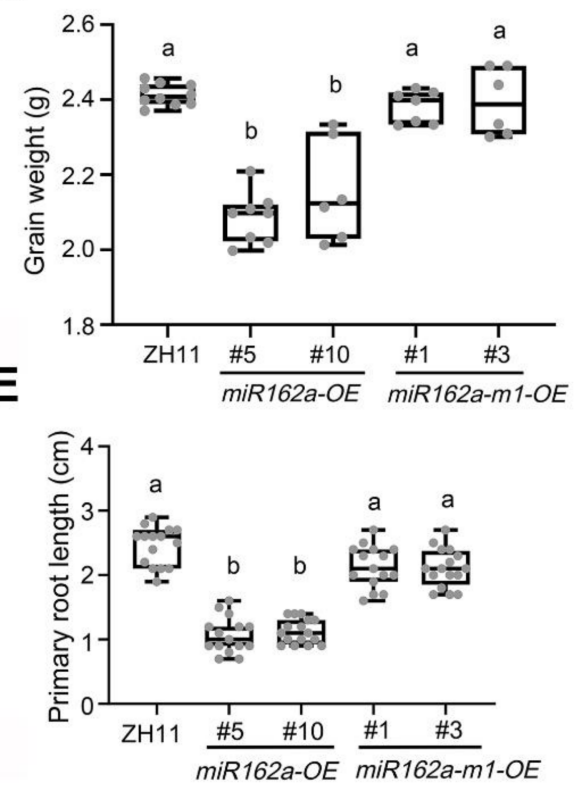

G

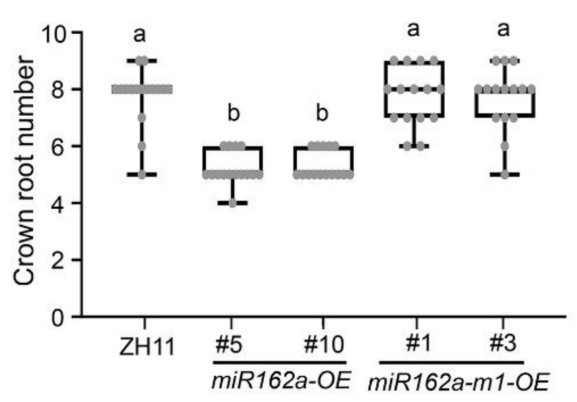

Figure 4. Overexpression of osa-miR162a, but not osa-miR162a-m1, impairs root and grain development in rice. (A) Grain development is affected by overexpression (OE) of osa-miR162a, but not osa-miR162a-m1. Scale bar =1 cm. (B) Grain width ranges of 16 randomly selected seeds of indicated genotypes. (C) Weight of 100 grains of indicated genotypes. At least six randomly pooled seeds (100 per pool) were evaluated for each genotype. (D) Primary root length of three-day-old seedlings is affected by overexpression of osa-miR162a, but not osa-miR162a-m1. Scale bar $=0.5 \mathrm{~cm}$. (E) Primary root length quantified using 15 randomly selected seedlings. (F) Crown root number of seven-day-old seedlings is affected by overexpression of osa-miR162a, but not osa-miR162a-m1. Scale bar $=0.5 \mathrm{~cm}$. (G) Quantification of the crown root number using 15 randomly selected seedlings. In (B,C,E,G), measurements are plotted as boxes and whiskers displaying mean \pm SD. Individual data points are shown as dots. Significant differences were determined using one-way ANOVA followed by Tukey post hoc test. Letters indicate significantly different groups. 
A

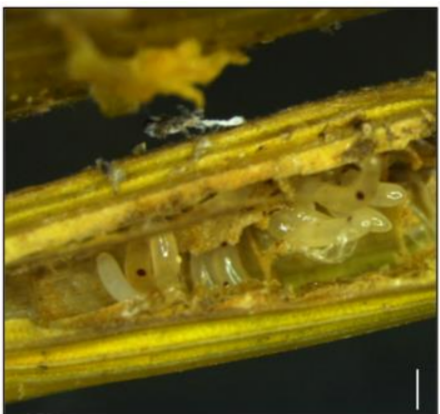

ZH11

B

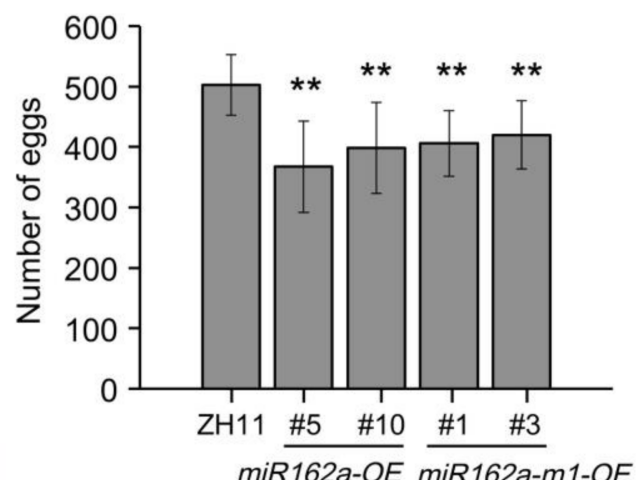

D

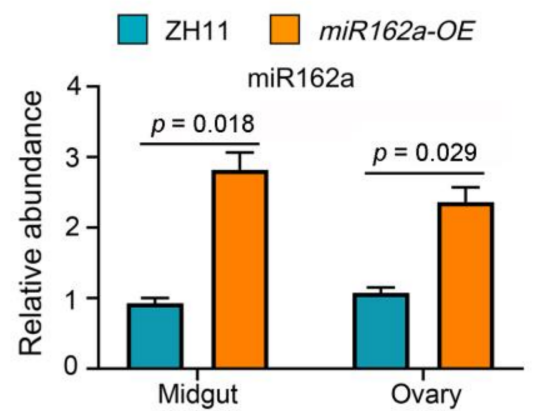

$\mathbf{F}$

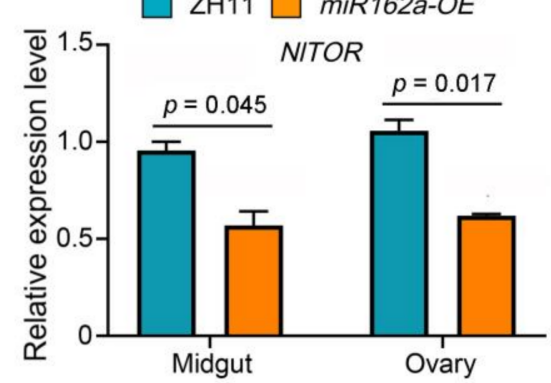

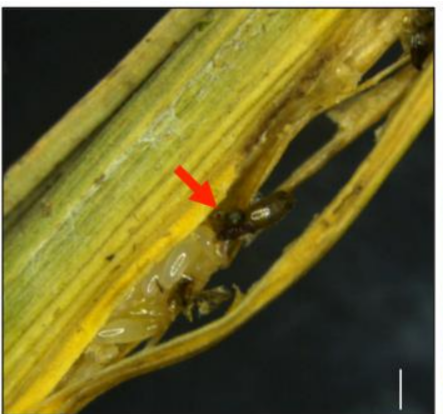

miR162a-OE

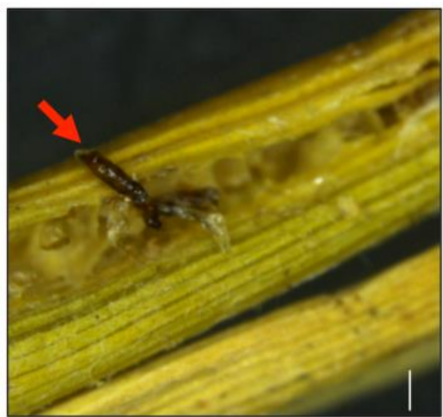

miR162a-m1-OE

C

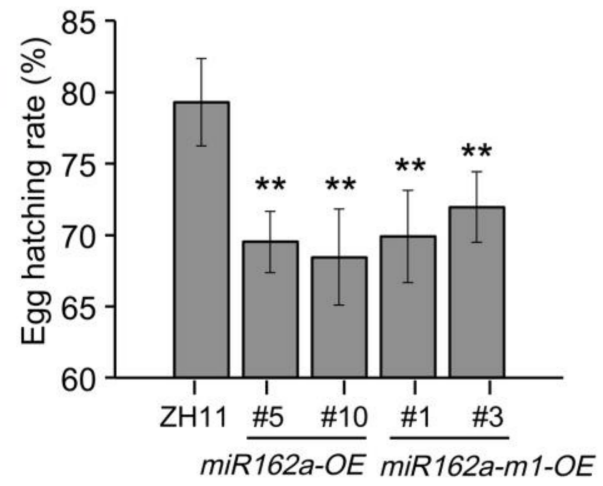

E

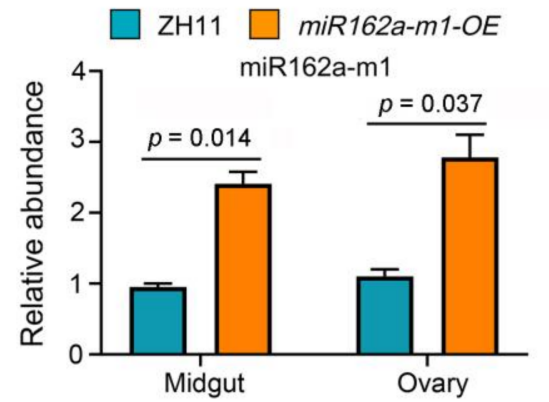

G

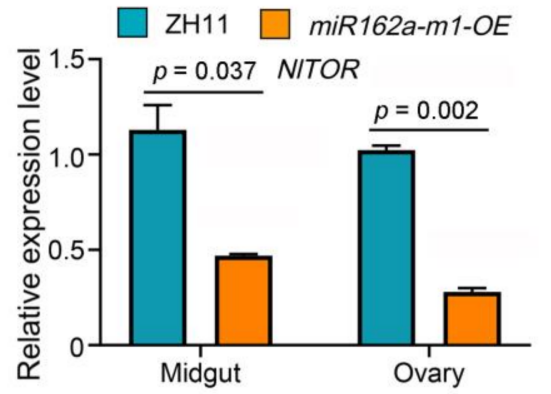

Figure 5. BPH insects fed with miR162a-OE or miR162a-m1-OE plants show impaired fecundity. (A) Insects fed with miR162a-OE or miR162a-m1-OE plants produce abnormal black eggs or eggs without pigmented eyes. Red arrows indicate black eggs. Scale bar $=0.5 \mathrm{~mm}$. (B,C) Quantification of egg numbers (B) and hatching rates (C) for insects fed with indicated genotypes. Eggs from eight female adults were evaluated and the data are presented as mean \pm SD. ${ }^{* *} p<0.01$ (Student's $t$-test). (D,E) Increased abundance of osa-miR162a (D) or osa-miR162a-m1 (E) in the midgut and ovary of insects fed with indicated genotypes. The miRNA abundances were determined by stem-loop RT-qPCR and the data are presented as mean \pm SEM. $p$ values are shown (Student's $t$-test). $(\mathbf{F}, \mathbf{G})$ Decreased NITOR expression in the midgut and ovary of insects fed with $m i R 162 a-O E(\mathbf{F})$ or miR162a-m1-OE (G) plants. The expression level was determined by RT-qPCR and the data are presented as mean \pm SEM. $p$ values are shown (Student's $t$-test). Two biological replicates were conducted and the miRNA or NITOR transcript abundance in insects fed with ZH11 plants was normalized as 1. 
To examine whether the observed fecundity defects of $\mathrm{BPH}$ were associated with the silencing of NITOR via the cross-kingdom RNAi, we interrogated BPH female adults continuously fed with miR162a-OE or miR162a-m1-OE plants. By stem-loop RT-qPCR, we detected relatively higher abundances of osa-miR162a or osa-miR162a-m1 in the midgut and ovary of female adults fed with miR162a-OE or miR162a-m1-OE plants than those fed with ZH11 plants (Figure 5D,E). Accordingly, the transcript levels of NITOR in the midgut and ovary of female adults fed with miR162a-OE or miR162a-m1-OE plants were considerably lower than those fed with ZH11 plants (Figure 5F,G). These results suggested that both osa-miR162a and osa-miR162a-m1 are capable of weakening the fecundity of BPH by silencing NITOR via the cross-kingdom RNAi.

Finally, we performed a host choice test between miR162a-OE, miR162a-m1-OE, and ZH11 plants at the young seedling stage. At $48 \mathrm{~h}$ after infestation, fewer insects were found to settle on miR162a-OE and miR162a-m1-OE plants than on ZH11 plants (Figure 6A). The survival rates of both miR162a-OE and miR162a-m1-OE seedlings were substantially higher than those of $\mathrm{ZH} 11$ plants (Figure 6B,C). These findings indicated that overproduction of osa-miR162a or osa-miR162a-m1 can confer rice resistance to BPH.
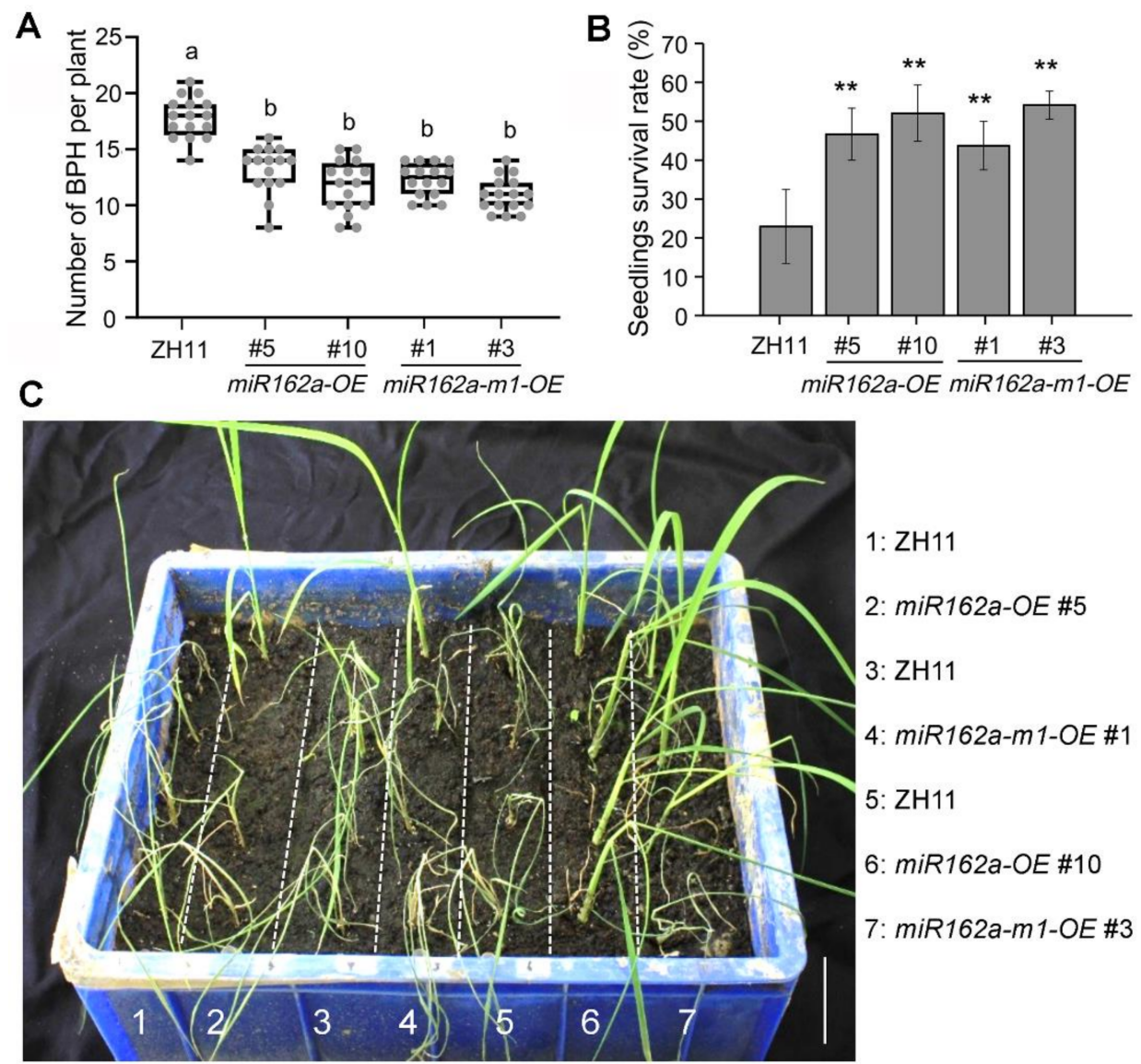

Figure 6. Overexpression of osa-miR162a or osa-miR162a-m1 confers rice resistance to BPH. (A) Number of insects settled on indicated genotypes at $48 \mathrm{~h}$ after infestation in a host choice test. Fifteen four-leaf-stage young seedlings were infested with 15 nymphs of the third instar per plant. Measurements are plotted as boxes and whiskers displaying mean \pm SD. Individual data points are shown as dots. Significant differences were determined using one-way ANOVA followed by Tukey post hoc test. Letters indicate significantly different groups. (B) Plant survival rates of indicated genotypes at 10 days after infestation. The data are presented as mean \pm SD of three biological replicates $(n=3) .{ }^{* *} p<0.01$ (Student's $t$-test). (C) Representative image of one replicate for $(\mathbf{B})$. Scale bar $=4 \mathrm{~cm}$. 


\section{Discussion}

In plants, miRNAs act as critical post-transcriptional regulators of gene expression in a broad range of biological processes, including responses to biotic stresses. Many rice miRNAs have been demonstrated to modulate plant immunity against pathogens or insect herbivores by fine-tuning the expression of endogenous target genes [22,23,38,39]. Notably, unlike animal miRNAs, plant miRNAs are 2'-O-methylated at the $3^{\prime}$ end, which is crucial for their increased stability [40]. Some plant miRNAs, as exemplified by miR159 and miR2911, have been reported to be extremely stable even under harsh conditions, such as acidic $\mathrm{pH}$ and cooking temperatures [41-43]. In the past decade, there has been a growing body of evidence showing that plant miRNAs, partially due to their superb stability, can enter an interacting organism from a different kingdom, such as fungus, insect, or mammal. In that organism, plant miRNAs can mediate the so-called crosskingdom RNAi to regulate its gene expression and reshape its biological activity, including development, reproduction, and virulence [18-21]. These groundbreaking discoveries have refreshed our understanding of the functions of plant miRNAs and opened up a new research area in miRNA biology. In this study, we found that intake of rice osa-miR162a mimics by feeding or injection dramatically down-regulated the expression of NITOR in $\mathrm{BPH}$ and consequently inhibited the TOR signaling-dictated Vg activity, ultimately leading to impaired fecundity (Figures 1C and 2B-D). Consistently, the BPH adults fed with transgenic rice plants overexpressing osa-miR162a exhibited ovipositing and hatching defects (Figure 5A-C). These results imply that rice osa-miR162a is a previously unknown player for defending BPH via the cross-kingdom RNAi mechanism. Intriguingly, the cole bra-miR162a has also been shown to regulate the caste development in honeybee through the cross-kingdom RNAi [18]. These two studies suggest that the insect TOR gene is a conserved target for plant miR162a during plant-insect interactions. It awaits further investigation whether plant miR162a has additional target genes in these insects.

There are two predicted osa-miR162a target sites in the mRNA of NITOR. The primary one is located in the CDS with 14 bases matching, while the secondary one resides in the $3^{\prime}$ UTR with 10 bases matching (Figure 1B). It was thought that animal miRNAs tend to bind the $3^{\prime}$ UTR of the target mRNA, whereas plant miRNAs predominately associate with the CDS [44]. However, more in-depth studies have suggested that both animal and plant miRNAs can bind to the $5^{\prime}$ UTR, $3^{\prime}$ UTR, or CDS of the target transcripts $[45,46]$. Unlike plant miRNAs, which generally require a perfect or near-perfect complementarity to their target mRNAs, most animal miRNAs exhibit an overall imperfect base pairing with their target mRNAs, except for the "seed region" at the $3^{\prime}$ end of the target sequence. It is also believed that not only the miRNA-target complementarity but also the target accessibility are major determinants for efficient miRNA-target interactions [47,48]. Due to these complexities, we are currently unable to distinguish which predicted target site of NITOR is effectively bound by osa-miR162a inside BPH. Notably, the cole bra-miR162a target site has been demonstrated to be located in the CDS of AmTOR in honeybee [18].

The modification of osa-miR162a to osa-miR162a-m1 simultaneously improved the complementarity to the NITOR mRNA at both the primary target site (i.e., from 14 base matches to 16) and the seed region of the secondary target site (i.e., from 6 base matches to 7 ) (Figure 3C). Therefore, we have anticipated that overexpression of osa-miR162a-m1 would exert a stronger effect than osa-miR162a on weakening the fecundity of BPH. However, we observed comparable reduction in the fecundity of $\mathrm{BPH}$ adults fed with transgenic rice plants overexpressing either osa-miR162a or osa-miR162a-m1, suggesting that the complementarity between osa-miR162a and NITOR is not the limiting factor to determine the effectiveness of osa-miR162a-mediated cross-kingdom RNAi.

Although overexpression of osa-miR162a promises an effective and economical means for $\mathrm{BPH}$ control in various rice cultivars, the overexpression negatively affected root development and grain production in rice (Figure 4). We found that osa-miR162a could potentially target eight endogenous genes in rice (Figure $3 \mathrm{~A}, \mathrm{~B})$, six of which were experimentally validated due to remarkable transcript reduction in miR162a-OE plants (Figure 3D). Little 
is known about the functions of these genes except OsDCL1 encoding a key enzyme for miRNA biosynthesis in rice. Strong silencing of OsDCL1 by over $90 \%$ resulted in growth arrest at the seedling stage [49]. Surprisingly, the OsDCL1 expression was suppressed by approximately 35 and 30\% upon overexpression of osa-miR162a or osa-miR162-m1, respectively (Figure 3D). Of particular note, OsDCL1 was not considered as a target gene of osa-miR162-m1 by WMD (Figure 3A), as there are six mismatches between them (Figure S3). We have previously characterized a large number of plant artificial miRNAs (amiRNAs) and found that effective plant amiRNAs often must contain less than two mismatches to the target site [47]. Therefore, the silencing of OsDCL1 by osa-miR162-m1 was exceptionally interesting. However, since overexpression of osa-miR162-m1 caused no developmental abnormality (Figure 4), OsDCL1 is unlikely to be the gene giving rise to the root or grain phenotype in transgenic rice plants overexpressing osa-miR162a. It warrants future studies to clarify which of the remaining genes is responsible for the observed root or grain defect.

In summary, this work reported the first cross-kingdom RNAi mechanism in rice-BPH interactions and revealed osa-miR162a and NITOR as the mobile rice miRNA and the BPH target gene, respectively, in this process. We also translated this finding into molecular breeding of BPH resistance in rice by overexpressing osa-miR162a, and further devised a widely useful strategy to retain the beneficial cross-kingdom RNAi effects but minimize undesirable perturbation on host gene expression for overexpressed plant miRNAs.

\section{Materials and Methods}

\subsection{Plant and Insect Materials}

The rice variety $\mathrm{ZH} 11$ was used as wild-type plants. Rice plants were routinely grown in a greenhouse with a cycle of $14 \mathrm{~h}$ light $\left(200 \mu \mathrm{mol} / \mathrm{m}^{2} / \mathrm{s}\right)$ at $28^{\circ} \mathrm{C}$ and $10 \mathrm{~h}$ dark at $25^{\circ} \mathrm{C}$. To compare the phenotypes of rice young seedlings, seeds harvested from miR162a-OE or miR162-m1-OE plants at the same time were surface-sterilized and germinated on $1 / 2$ Murashige \& Skoog agar medium in a growth chamber under the conditions described above. A laboratory strain of brown planthopper was obtained from the Guangdong Academy of Agricultural Sciences, China.

\subsection{Molecular Cloning and Transforming Rice Plants}

Routine molecular cloning procedures were followed for plasmid construction. To generate the osa-miR162a-m1 precursor, the osa-miR162a precursor was subjected to PCRbased site-directed mutagenesis. The precursor of osa-miR162a or osa-miR162a-m1 was inserted into the binary vector pCAMBIA1300 between AvrII and SacI restriction sites for overexpression. The binary vectors were transformed into Agrobacterium tumefaciens strain EHA105 cells through electroporation. Agrobacteria containing the pCAMBIA1300pre-miR162a or pCAMBIA1300-pre-miR162a-m1 plasmid were used for transforming rice callus. Transformed rice callus cells were selected by hygromycin resistance and regenerated into whole plants according to a standard protocol [50].

\subsection{Feeding BPH with Osa-miR162a Mimics}

For rearing on artificial diets, glass cylinders of $9.0 \mathrm{~cm}$ in length and $2.0 \mathrm{~cm}$ in diameter were used as feeding chambers. The artificial diet was coated with synthetic osa-miR162a mimics (RIBBIO, Guangzhou, China) to a final concentration of $100 \mathrm{ng} / \mu \mathrm{L}$, and then placed between two layers of stretched Parafilm that were located at two open ends of the chamber. The artificial diets were renewed every day. The chambers were covered with a piece of black cotton cloth, and the two ends with artificial diets were exposed to light. The insects could eat by puncturing the inner Parafilm layer of the food pouch. Ten female adults of the first day were placed in each chamber, and sets of 100 individuals were considered as a biological replicate in each group. Three replicates were performed. The midgut, fat body, hemolymph, and other tissues from the female adults after 24,48 , and $72 \mathrm{~h}$ feeding were dissected in insect saline containing $0.75 \% \mathrm{NaCl}$ and stored at $-80^{\circ} \mathrm{C}$ for further use. 


\subsection{Injecting BPH with Osa-miR162a Mimics}

Female adults of the first day were used for the injection experiment. The osa-miR162a mimics of $50 \mathrm{ng}(1 \mathrm{ng} / \mathrm{nL})$ were injected into the side of the abdomen of the nymph using a NARISHIGE IM-31 microinjector (Nikon, Tokyo, Japan). A control was performed using an equivalent amount of negative control miRNA mimics. Sets of 100 individuals were considered as a biological replicate in each group. Ten individuals were set for a group for 24,48 , and $72 \mathrm{~h}$ sampling. Three replicates were performed.

\subsection{Bioassay of the Fecundity}

In the experiments evaluating the fecundity of BPH, each newly emerged female adult after injection with osa-miR162a mimics was transferred to a fresh rice plant, and single paired with an untreated male adult. Controls were performed in which an equivalent volume of negative control miRNA mimics was injected into the females. The treatment group and control group each included 20 pairs of adults. After the eggs were hatched, the newly hatched nymphs were counted and removed every day. Then, rice was dissected and counted under the microscope 15 days later. We summed the numbers of hatched nymphs and unhatched eggs as the total number of eggs. The hatching rate was calculated by dividing the number of hatched nymphs against the total number of eggs. Eggs with no sign of development or completely developed eggs containing no nymphs were considered as unhatched eggs.

\subsection{RT-qPCR or Stem-Loop RT-qPCR}

Total RNA was isolated from BPH female adults or rice using the TRIzol reagent (Invitrogen, CA, USA). Regular or stem-loop RT-qPCR was performed as previously described [51]. Briefly, total RNA was converted into the first-strand cDNA with stem-loop RT primers for osa-miR162a or osa-miR162a-m1 or with an oligo dT primer for other genes using the PrimeScript RT reagent Kit plus genomic DNA Eraser (TaKaRa, Tokyo, Japan). The qPCR was performed in a LightCycler 96 Instrument (Roche, Indianapolis, IN, USA) using the SYBR ${ }^{\circledR}$ Premix Ex TaqTM Kit (TaKaRa, Tokyo, Japan). The relative miRNA or transcript abundance was normalized to the housekeeping gene Os18s (18S rRNA, Os09g00999) in rice or NlGAPDH (Glyceraldehyde-3-phosphate dehydrogenase) in BPH. The qPCR primers used in this study are listed in Supplementary Table S1.

\subsection{Feeding BPH with miR162a-OE or miR162a-m1-OE Plants}

Five pregnant female adults feeding on ZH11 plants were transferred to fresh ZH11, miR162a-OE, or miR162a-m1-OE plants. After continuous feeding from parents to offspring adults, the midgut and ovary tissues from 10 pregnant female adults were dissected in insect saline containing $0.75 \% \mathrm{NaCl}$. Newly hatched $\mathrm{BPH}$ nymphs were fed with miR162a$O E$ or miR162a-m1-OE plants. As they grew up, a pair of female and male adults were mated, and the fecundity was analyzed as described above.

\subsection{BPH Host Choice Test}

Eight four-leaf-stage young seedlings of ZH11, miR162a-OE or miR162a-m1-OE were grown in the same plastic bucket $(20 \mathrm{~cm}$ in length, $15 \mathrm{~cm}$ in width, and $15 \mathrm{~cm}$ in height). About $300 \mathrm{BPH}$ nymphs of the third instar were released in the bucket and allowed to choose a host plant. The insects settled on each plant were counted at $48 \mathrm{~h}$ after infestation. The survival rate of rice seedlings was determined at the indicated time. Two biological replicates were conducted.

\subsection{Bioinformatic Prediction of miRNA Target Genes}

The target sites of osa-miR162a or osa-miR162a-m1 in NITOR were predicted using the algorithms RNAhybrid (https:/ / bibiserv.cebitec.uni-bielefeld.de/rnahybrid, accessed on 19 November 2021) [52] and miRanda (http:/ / cbio.mskcc.org/miRNA2003/miranda.html, accessed on 19 November 2021) [53]. To predict target genes of osa-miR162a or osa-miR162a- 
$\mathrm{m} 1$ in rice, the WMD was used (http: / / wmd3.weigelworld.org/, accessed on 19 November 2021) [54]. In the target search program interface of WMD, the 21-nt osa-miR162a or osamiR162a-m1 sequence was inputted and searched against the database Oryza sativa cDNA v6.1, which has been integrated into the program, with the base mismatch setting as five or less.

\subsection{Statistical Analysis}

All the standard statistical analyses were conducted using GraphPad Prism 8.0 software. For multiple comparisons, one-way of variance (ANOVA) with post hoc Tukey tests were used. For two-sample unpaired comparisons, Student's $t$-tests were used.

\section{Patents}

The data of the current research have applied for a China invention patent (application number: ZL202110873616X).

Supplementary Materials: The following are available online at https:/ / www.mdpi.com/article / 10.3390/ijms222312652/s1.

Author Contributions: J.-F.L., W.Z. and J.C. conceived and supervised the research. W.S. performed most of the experiments, including the construction of transgenic rice, phenotypic analysis, and resistance analysis of rice to BPH. S.C. and J.L. evaluated the effect of miR162a mimics on BPH. J.-F.L., W.S. and J.C. wrote the manuscript. All authors reviewed, revised, and approved the final manuscript. All authors have read and agreed to the published version of the manuscript.

Funding: This research was funded by the Foundation of Guangzhou Science and Technology Key Project (201904020041) and the National Natural Science Foundation of China (31972262).

Institutional Review Board Statement: Not applicable.

Informed Consent Statement: Not applicable.

Data Availability Statement: All relevant data can be found within the manuscript or its Supplementary Materials on the journal website.

Acknowledgments: The authors thank the members of Jian-Feng Li's and Wenqing Zhang's laboratories for helpful discussion on this work.

Conflicts of Interest: The authors declare no conflict of interest except for the patent issue.

\section{References}

1. Lou, Y.G. Basic research on the outbreak mechanism and sustainable management of rice planthoppers. Chin. J. Appl. Entomol. 2011, 48, 231-238.

2. Ndiath, M.O. Insecticides and insecticide resistance. Methods Mol. Biol. 2019, 2013, 287-304.

3. Vaughn, T.; Cavato, T.; Brar, G.; Coombe, T.; DeGooyer, T.; Ford, S.; Groth, M.; Howe, A.; Johnson, S.; Kolacz, K.; et al. A method of controlling corn rootworm feeding using a Bacillus thuringiensis protein expressed in transgenic maize. Crop Sci. 2005, 45, 931-938. [CrossRef]

4. Tabashnik, B.E.; Gassmann, A.J.; Crowder, D.W.; Yves, C. Insect resistance to Bt crops: Evidence versus theory. Nat. Biotechnol. 2008, 26, 199-202. [CrossRef]

5. Pallava, B. Hardy cotton-munching pests are latest blow to GM crops. Science 2010, 327, 1439.

6. Sokol, N.S.; Xu, P.; Jan, Y.N.; Ambros, V. Drosophila let-7 microRNA is required for remodeling of the neuromusculature during metamorphosis. Genes Dev. 2008, 22, 1591-1596. [CrossRef]

7. Gomez-Orte, E.; Belles, X. MicroRNA-dependent metamorphosis in hemimetabolan insects. Proc. Natl. Acad. Sci. USA 2009, 106, 21678-21682. [CrossRef] [PubMed]

8. Ling, L.; Ge, X.; Li, Z.; Zeng, B.; Xun, J.; Aslam, A.F.M.; Song, Q.; Shang, P.; Huang, Y.; Tan, A. MicroRNA Let-7 regulates molting and metamorphosis in the silkworm, Bombyx mori. Insect Biochem. Mol. Biol. 2014, 53, 13-21. [CrossRef]

9. Belles, X. MicroRNAs and the evolution of insect metamorphosis. Annu. Rev. Entomol. 2017, 62, 111-125. [CrossRef]

10. Wu, W.; Xiong, W.; Li, C.; Zhai, M.; Li, Y.; Ma, F.; Li, B. MicroRNA-dependent regulation of metamorphosis and identification of microRNAs in the red flour beetle, Tribolium castaneum. Genomics 2017, 109, 362-373. [CrossRef] [PubMed]

11. Zhang, X.; Lu, K.; Zhou, Q. Dicer1 is crucial for the oocyte maturation of telotrophic ovary in Nilaparvata lugens (Stål) (Hemiptera: Geometroidea). Arch. Insect Biochem. Physiol. 2013, 84, 194-208. [CrossRef] 
12. Fu, X.; Li, T.; Chen, J.; Dong, Y.; Qiu, J.; Kang, K.; Zhang, W. Functional screen for microRNAs of Nilaparvata lugens reveals that targeting of glutamine synthase by miR-4868b regulates fecundity. J. Insect Physiol. 2015, 83, 22-29. [CrossRef] [PubMed]

13. Chen, J.; Liang, Z.; Liang, Y.; Pang, R.; Zhang, W.Q. Conserved microRNAs miR-8-5p and miR-2a-3p modulate chitin biosynthesis in response to 20-hydroxyecdysone signaling in the brown planthopper, Nilaparvata lugens. Insect Biochem. Mol. Biol. 2013, 43, 839-848. [CrossRef]

14. Li, T.; Chen, J.; Fan, X.; Chen, W.; Zhang, W.Q. MicroRNA and dsRNA targeting chitin synthase A reveal a great potential for pest management of the hemipteran insect Nilaparvata lugens. Pest Manag. Sci. 2017, 73, 1529-1537. [CrossRef] [PubMed]

15. Chen, J.; Li, T.; Pang, R.; Yue, X.Z.; Hu, J.; Zhang, W.Q. Genome-wide screening and functional analysis reveal that the specific microRNA nlu-miR-173 regulates molting by targeting Ftz-F1 in Nilaparvata lugens. Front. Physiol. 2018, 9, 1854. [CrossRef]

16. Agrawal, A.; Rajamani, V.; Reddy, V.S.; Mukherjee, S.K.; Bhatnagar, R.K. Transgenic plants over-expressing insect-specific microRNA acquire insecticidal activity against Helicoverpa armigera: An alternative to Bt-toxin technology. Transgenic Res. 2015, 24, 1-11. [CrossRef]

17. Jia, L.; Zhang, D.; Xiang, Z.; He, N. Nonfunctional ingestion of plant miRNAs in silkworm revealed by digital droplet PCR and transcriptome analysis. Sci. Rep. 2015, 5, 12290. [CrossRef] [PubMed]

18. Zhu, K.; Liu, M.; Fu, Z.; Zhou, Z.; Kong, Y.; Liang, H.; Lin, Z.; Luo, J.; Zheng, H.; Wan, P.; et al. Plant microRNAs in larval food regulate honeybee caste development. PLoS Genet. 2017, 13, e1006946. [CrossRef]

19. Zhang, L.; Hou, D.X.; Chen, X.; Li, D.; Zhu, L.; Zhang, Y.; Li, J.; Bian, Z.; Liang, X.; Cai, X.; et al. Exogenous plant MIR168a specifically targets mammalian LDLRAP1: Evidence of cross-kingdom regulation by microRNA. Cell Res. 2012, 22, 107-126. [CrossRef]

20. Wang, M.; Weiberg, A.; Lin, F.M.; Thomma, B.; Huang, H.D.; Jin, H.L. Bidirectional cross-kingdom RNAi and fungal uptake of external RNAs confer plant protection. Nat. Plants 2016, 2, 16151. [CrossRef]

21. Zhang, T.; Zhao, Y.L.; Zhao, J.H.; Wang, S.; Jin, Y.; Chen, Z.Q.; Fang, Y.Y.; Hua, C.L.; Ding, S.W.; Guo, H.S. Cotton plants export microRNAs to inhibit virulence gene expression in a fungal pathogen. Nat. Plants 2016, 2, 16153. [CrossRef] [PubMed]

22. Ge, Y.F.; Han, J.Y.; Zhou, G.X.; Xu, Y.; Ding, Y.; Shi, M.; Guo, C.; Wu, G. Silencing of miR156 confers enhanced resistance to brown planthopper in rice. Planta 2018, 248, 813-826. [CrossRef] [PubMed]

23. Dai, Z.; Tan, J.; Zhou, C.; Yang, X.; Yang, F.; Zhang, S.; Sun, S.; Miao, X.; Shi, Z. The OsmiR396-OsGRF8-OsF3H-flavonoid pathway mediates resistance to the brown planthopper in rice (Oryza sativa). Plant Biotechnol. J. 2019, 17, 1657-1669. [CrossRef]

24. Raikhel, A.S.; Dhadialla, T.S. Accumulation of yolk proteins in insect oocytes. Annu. Rev. Entomol. 1992, 37, 217-251. [CrossRef]

25. Greenberg, S.M.; Spurgeon, D.W.; Sappington, T.W.; Sétamou, M. Size-dependent feeding and reproduction by Boll weevil (Coleoptera: Curculionidae). J. Econ. Entomol. 2005, 98, 749-756. [CrossRef]

26. Tufail, M.; Naeemullah, M.; Elmogy, M.; Sharma, P.N.; Takeda, M.; Nakamura, C. Molecular cloning, transcriptional regulation, and differential expression profiling of vitellogenin in two wing-morphs of the brown planthopper, Nilaparvata lugens Stål (Hemiptera: Delphacidae). Insect Mol. Biol. 2010, 19, 787-798. [CrossRef]

27. Roy, S.G.; Raikhel, A.S. Nutritional and hormonal regulation of the TOR effector 4E-binding protein (4E-BP) in the mosquito Aedes aegypti. FASEB J. 2012, 26, 1334-1342. [CrossRef]

28. Hansen, I.A.; Attardo, G.M.; Park, J.H.; Peng, Q.; Raikhel, A.S. Target of rapamycin-mediated amino acid signaling in mosquito anautogeny. Proc. Natl. Acad. Sci. USA 2004, 101, 10626-10631. [CrossRef] [PubMed]

29. Qiu, J.; He, Y.; Zhang, J.; Kang, K.; Li, T.; Zhang, W. Discovery and functional identification of fecundity-related genes in the brown planthopper by large-scale RNA interference. Insect Mol. Biol. 2016, 25, 724-733. [CrossRef]

30. Gulia-Nuss, M.; Robertson, A.E.; Brown, M.R.; Strand, M.R. Insulin-like peptides and the target of rapamycin pathway coordinately regulate blood digestion and egg maturation in the mosquito Aedes aegypti. PLoS ONE 2011, 6, e20401.

31. Lu, K.; Chen, X.; Liu, W.T.; Zhou, Q. TOR pathway-mediated juvenile hormone synthesis regulates nutrient-dependent female reproduction in Nilaparvata lugens (Stål). Int. J. Mol. Sci. 2016, 17, 438. [CrossRef] [PubMed]

32. Liu, F.; Li, K.; Cai, W.; Zhao, J.; Zou, Y.; Hua, H. Knockdown of TOR causing ovarian diapause in a genetically stable brachypterous strain of Nilaparvata lugens. Arch. Insect Biochem. Physiol. 2017, 95, e21400. [CrossRef]

33. Zhuo, J.C.; Xue, J.; Lu, J.B.; Huang, H.; Xu, H.; Zhang, C. Effect of RNAi-mediated knockdown of NlTOR gene on fertility of male Nilaparvata lugens. J. Insect Physiol. 2017, 98, 149-159. [CrossRef] [PubMed]

34. Park, J.H.; Attardo, G.M.; Hansen, I.A.; Raikhel, A.S. GATA factor translation is the final downstream step in the amino acid/target-of-rapamycin-mediated vitellogenin gene expression in the anautogenous mosquito Aedes aegypti. J. Biol. Chem. 2006, 281, 11167-11176. [CrossRef]

35. Tatebe, H.; Shiozaki, K. Evolutionary conservation of the components in the TOR signaling pathways. Biomolecules 2017, 7, 77. [CrossRef] [PubMed]

36. Varkonyi-Gasic, E.; Wu, R.; Wood, M.; Walton, E.; Hellens, R.P. Protocol: A highly sensitive RT-PCR method for detection and quantification of microRNAs. Plant Methods 2007, 3, 12. [CrossRef]

37. Li, X.; Ma, X.; Wang, H.; Zhu, Y.; Liu, X.; Li, T.; Zheng, Y.P.; Zhao, J.Q.; Zhang, J.W.; Huang, Y.Y.; et al. Osa-miR162a fine-tunes rice resistance to Magnaporthe oryzae and yield. Rice 2020, 13, 38. [CrossRef] [PubMed]

38. Li, Y.; Cao, X.L.; Zhu, Y.; Yang, X.M.; Zhang, K.N.; Xiao, Z.Y.; Wang, H.; Zhao, J.H.; Zhang, L.L.; Li, G.B.; et al. Osa-miR398b boosts $\mathrm{H}_{2} \mathrm{O}_{2}$ production and rice blast disease-resistance via multiple superoxide dismutases. New Phytol. 2019, 222, 1507-1522. [CrossRef] 
39. Wang, H.; Li, Y.; Chern, M.; Zhu, Y.; Zhang, L.; Lu, J.; Li, X.P.; Dang, W.Q.; Ma, X.C.; Yang, Z.R.; et al. Suppression of rice miR168 improves yield, flowering time and immunity. Nat. Plants 2021, 7, 129-136. [CrossRef]

40. Yu, B.; Yang, Z.; Li, J.; Minakhina, S.; Yang, M.; Padgett, R.W.; Steward, R.; Chen, X. Methylation as a crucial step in plant microRNA biogenesis. Science 2005, 307, 932-935. [CrossRef]

41. Jung, M.; Schaefer, A.; Steiner, I.; Kempkensteffen, C.; Stephan, C.; Erbersdobler, A.; Jung, K. Robust MicroRNA stability in degraded RNA preparations from human tissue and cell samples. Clin. Chem. 2010, 56, 998-1006. [CrossRef] [PubMed]

42. Zhou, Z.; Li, X.; Liu, J.; Dong, L.; Chen, Q.; Liu, J.; Kong, H.; Zhang, Q.; Qi, X.; Hou, D.; et al. Honeysuckle-encoded atypical microRNA2911 directly targets influenza A viruses. Cell Res. 2015, 25, 39-49. [CrossRef]

43. Chin, A.R.; Fong, M.Y.; Somlo, G.; Wu, J.; Swiderski, P.; Wu, X.; Swiderski, P.; Wu, X.; Wang, S.E. Cross-kingdom inhibition of breast cancer growth by plant miR159. Cell Res. 2016, 26, 217-228. [CrossRef]

44. Brodersen, P.; Sakvarelidze-Achard, L.; Bruun-Rasmussen, M.; Dunoyer, P.; Yamamoto, Y.Y.; Sieburth, L.; Voinnet, O. Widespread translational inhibition by plant miRNAs and siRNAs. Science 2008, 320, 1185-1190. [CrossRef] [PubMed]

45. Brousse, C.; Liu, Q.; Beauclair, L.; Deremetz, A.; Axtell, M.J.; Bouche, N. A non-canonical plant microRNA target site. Nucleic Acids Res. 2014, 42, 5270-5279. [CrossRef] [PubMed]

46. Seok, H.; Ham, J.; Jang, E.S.; Chi, S.W. MicroRNA target recognition: Insights from transcriptome-wide non-canonical interactions. Mol. Cells 2016, 39, 375-381.

47. Li, J.-F.; Chung, H.; Niu, Y.; Bush, J.; McCormack, M.; Sheen, J. Comprehensive protein-based artificial microRNA screens for effective gene silencing in plants. Plant Cell 2013, 25, 1507-1522. [CrossRef]

48. Li, J.; Reichel, M.; Millar, A.A. Determinants beyond both complementarity and cleavage govern MicroR159 efficacy in Arabidopsis. PLoS Genet. 2014, 10, e1004232. [CrossRef] [PubMed]

49. Liu, B.; Li, P.; Li, X.; Liu, C.; Cao, S.; Chu, C.; Cao, X. Loss of function of OsDLC1 affects microRNA accumulation and causes developmental defects in rice. Plant Physiol. 2005, 139, 296-305. [CrossRef]

50. Hiei, Y.; Ohta, S.; Komari, T.; Kumashiro, T. Efficient transformation of rice (Oryza sativa L.) mediated by Agrobacterium and sequence analysis of the boundaries of the T-DNA. Plant J. 1994, 6, 271-282. [CrossRef]

51. Zhang, N.; Zhang, D.; Shen, W.; Li, J.-F. Engineered artificial microRNA precursors facilitate cloning and gene silencing in Arabidopsis and Rice. Int. J. Mol. Sci. 2019, 20, 5620. [CrossRef] [PubMed]

52. Krüger, J.; Rehmsmeier, M. RNAhybrid: MicroRNA target prediction easy, fast and flexible. Nucleic Acids Res. 2006, 34, W451-W454. [CrossRef]

53. Enright, A.J.; John, B.; Gaul, U.; Tuschl, T.; Sander, C.; Marks, D.S. MicroRNA targets in Drosophila. Genome Biol. 2003,5 , R1. [CrossRef] [PubMed]

54. Schwab, R.; Ossowski, S.; Riester, M.; Warthmann, N.; Weigel, D. Highly specific gene silencing by artificial microRNAs in Arabidopsis. Plant Cell 2006, 18, 1121-1133. [CrossRef] [PubMed] 